\title{
Nanophysiology: fact or fiction?
}

\author{
Hans Oberleithner
}

Received: 15 January 2008 / Accepted: 18 January 2008 /Published online: 1 February 2008

(C) Springer-Verlag 2008

When in 1982 the scanning tunneling microscope was invented [1], its possibilities excited those scientists working in the material sciences. Electrically conductive material could be investigated with such precision that physics moved into a new nanoscale era. When in 1986 the principle of the atomic force microscope was described [2] and Binnig and Rohrer, the inventors of the tunneling microscope were awarded the Nobel prize for physics, life scientists paid little attention. At that time, molecular biology and molecular genetics were starting their logarithmic phase of development but, in addition, there was a small and heterogenous community of scientists with a major interest in biology who dared to take the first steps in applying these new technological advances to biology. Although there were a number of exciting breakthroughs in the 1990s, most life scientists at that time followed the rapid developments of cell biology, but there appeared to be little intellectual curiosity for other lines of enquiry.

Toward the end of the 1990s, however, the life science community started to realize the potential of atomic force microscopy (AFM). Researchers slowly came to the conclusion that AFM is not simply another microscope that, at best, can compete with the well-established electron microscope but that it is a versatile instrument - an invasive tool, a scanning microscope, a force measuring devicethat allows novel approaches in the study of live cells.

Fortunately, physicists and, in particular, biophysicists helped to pave the way for making the AFM a tool suitable for biologists, physiologists, and medical scientists. At the

H. Oberleithner $(\bowtie)$

Westfaelische Wilhelms University, Institute of Physiology II,

Robert-Koch-Str. 27 B,

Innenhof, Germany

e-mail: oberlei@uni-muenster.de same time, some industrial companies realized the potential of the AFM as a new research tool and produced AFMs easy to use for studying biological samples, from living cells to single molecules.

Now, at the beginning of 2008, the ice is broken and the wide field of cellular and molecular physiology is ready for AFM. This was what drove us to collect 21 papers from laboratories around the world that use AFM as their major research tool. The selection of contributors to this special issue named "Nanophysiology" was made on the basis of AFM expertise and a clear commitment to approach open questions of physiology. The common denominator was 'AFM,' but the physiological window was wide open.

In this special issue, there are about the same number of reviews and original papers. The reviews focus on different topics. They should help to understand why AFM is useful for life science research, how different AFM modes are applied, and what is novel about such approaches. It is hoped that the reviews will also help to calm down prejudices that AFM is a technique that is too complex and difficult to handle and expensive to run, whereas today none of these is true.

Intermingled between the reviews, there are original papers that demonstrate that important questions in physiology, biology, and medicine can be addressed in this unique way. In addition to high-resolution imaging at the nanoscale level, a major emphasis is put on cellular and molecular mechanics by using native live material that has not been treated with fixatives. This has enabled the stiffness of living cells to be quantitatively measured - a new parameter in cell physiology. Eventually, mechanical cell membrane stiffness may be recognized to be as important as the electrical cell membrane potential, one of the major trademarks in physiology. I would suggest that in a few years membrane, stiffness may be another. 
Finally, this special issue demonstrates that AFM is not a high-end technology, the limits of which are already clearly visible. Unexpected progress in terms of scan speed, spatial resolution, and specific molecule recognition is taking place. There is also progress in combining AFM with other techniques such as fluorescence microscopy, AFM single molecule recognition, molecule adhesion, and elasticity in living matter. Taken together, the atomic force microscope is a tool that quantifies physically obtained parameters of cells and molecules in nanometers.
As the title of this introduction suggests "nanophysiology" is progressing from fiction to fact. AFM will certainly facilitate this process.

\section{References}

1. Binnig G, Rohrer H (1982) Scanning tunneling microscopy. Helvetica Physica Acta 55:726-735

2. Binnig G, Quate CF, Gerber C (1986) Atomic force microscope. Phys Rev Letters 56:930-933 